9 Tilg H, Jalan R, Kaser A et al. Anti-tumor necrosis factor-alpha monoclonal antibody therapy in severe alcoholic hepatitis. J Hepatol 2003; 38:419-25.

10 Ostuni P, Botsios C, Punzi L et al. Hepatitis B reactivation in a chronic hepatitis B surface antigen carrier with rheumatoid arthritis treated with infliximab and low dose methotrexate. Ann Rheum Dis $2003 ; 62: 686-7$

Conflicts of interest: none declared.

\section{Paraneoplastic pemphigus associated with follicular dendritic cell sarcoma and Castleman disease}

DOI: $10.1111 / j .1365-2133.2005 .06695 . x$

SIR, Paraneoplastic pemphigus (PNP) is an autoimmune blistering and erosive mucocutaneous disease associated with neoplasia, most commonly of lymphoreticular origin, first described by Anhalt et al. ${ }^{1}$ An alternative term, paraneoplastic autoimmune multiorgan syndrome, has since been proposed to define a condition in which patients, in addition to severe, often fatal pulmonary involvement and deposition of autoantibodies in different organs, may display lesions that resemble pemphigoid, erythema multiforme, graft-versus-host-disease and lichen planus as well as classic pemphigus. ${ }^{2}$ We report a patient with a lichen planus-like mucocutaneous variant of PNP associated with follicular dendritic cell (FDC) sarcoma and Castleman disease (CD), emphasizing in particular the rarity of PNP in association with such a type of sarcoma.

A 53-year-old woman had painful persistent oral erosions accompanied by dry cough, dysphagia and weight loss since December 2002. She had been given topical and systemic corticosteroids at another hospital, with no improvement. In June 2003, when the patient was referred to our institute, she presented with extensive erosions of the oral mucosa (Fig. 1a), tongue and conjunctiva and a 3-week history of lichenoid papules symmetrically distributed on the trunk (Fig. 1b) and extremities. Laboratory examinations revealed mild anaemia (haemoglobin $10 \cdot 7 \mathrm{~g} \mathrm{dL}^{-1}$; normal 12-16) and positive antinuclear antibodies with speckled pattern at a titre of $1: 160$. Histology from a papular area showed a lichenoid interface dermatitis with numerous necrotic keratinocytes (Fig. 2a). Direct immunofluorescence disclosed intercellular deposits of IgG throughout the epidermis, whereas indirect immunofluorescence demonstrated IgG autoantibodies directed to the intercellular substance of stratified epithelium, namely monkey oesophagus, and also of rat bladder transitional cell epithelium. An immunoprecipitation analysis, carried out as reported, ${ }^{3}$ identified a complex of three antigens: proteins of 210 and $190 \mathrm{kDa}$ that could represent desmoplakin II/envoplakin and periplakin, respectively, and the as yet uncharacterized 170-kDa antigen (Fig. 2b). A specific enzyme-linked immuno-
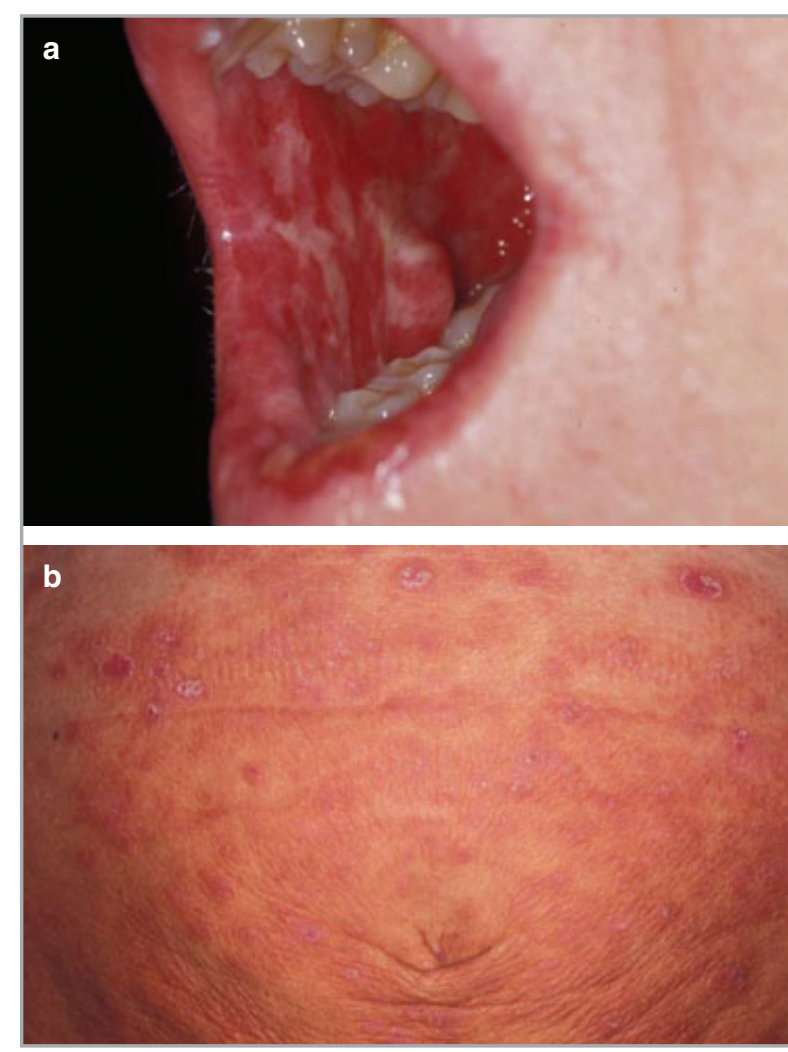

Fig 1. (a) Erosions and pseudomembranes in the oral mucosa and lips. (b) Lichenoid papules coalescing into plaques over the abdomen.

sorbent assay, using recombinant desmoglein (Dsg) 1 and Dsg3 proteins, ${ }^{4}$ did not detect antibodies to either Dsg1 or Dsg3. A diagnosis of PNP was made and a search for an underlying neoplasm revealed, on abdominal computed tomographic scans, a $9 \times 5 \times 8 \mathrm{~cm}$ soft tissue mass in the right retroperitoneal area. The mass was excised and pathological examination showed histological features of FDC sarcoma in association with residual foci of $\mathrm{CD}$ of the hyaline-vascular type. On immunohistochemistry, the tumour cells of the sarcoma had a $\mathrm{CD} 21+$, CD35+, nerve growth factor receptor-positive, actinpositive phenotype. Within 3 weeks after resection of the tumour the mucocutaneous manifestations continued to deteriorate and therapy was initiated with intravenous methylprednisolone $80 \mathrm{mg}$ daily and azathioprine $100 \mathrm{mg}$ daily for 15 days, but this was ineffective. Pulsed intravenous methylprednisolone $250 \mathrm{mg}$ daily for 5 days, and subsequently, a cycle of high-dose intravenous immunoglobulins $0.6 \mathrm{~g} \mathrm{~kg}^{-1}$ daily for five consecutive days resulted in moderate clinical improvement. However, she developed ingravescent dyspnoea: respiratory function tests disclosed restrictive pulmonary changes, whereas positron emission tomography of the chest showed features suggestive of secondary lung involvement by sarcoma. She died of respiratory failure in April 2004.

In our patient, the association of PNP with FDC sarcoma, a rare malignant neoplasm of follicular dendritic cells, is unique. Notably, the FDC sarcoma arose from CD, as seen in only one previously reported case. ${ }^{5}$ Although there is no firm evidence 
Fig 2. (a) Photomicrograph showing a lichenoid interface dermatitis with necrotic keratinocytes (haematoxylin and eosin; original magnification $\times 200$ ).

(b) Immunoprecipitation of ${ }^{35}$ S-labelled keratinocyte extracts by patient and control sera. Patient serum reacted with three bands at 210,190 and $170 \mathrm{kDa}$. The 250-kDa antigen, not immunoprecipitated by patient serum.

Lane 1, patient serum; lane 2, normal human serum; lane 3 , positive control from a patient with known paraneoplastic pemphigus. The bars on the right side indicate molecular mass standards at 220, 110 and $98 \mathrm{kDa}$. detected by the positive control serum, was

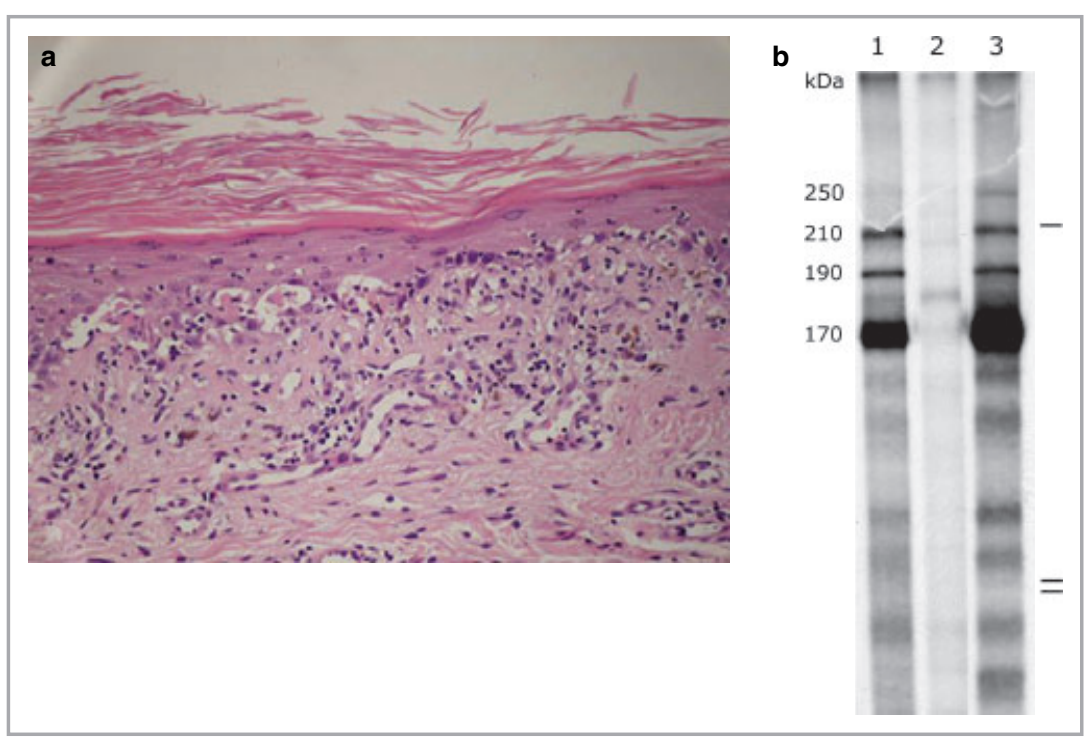

\section{References}

of the former as a possible precursor of the latter has been suggested: the FDC tumour presumably develops through a hyperplasia-dysplasia-neoplasia sequence, as documented for some epithelial neoplasms. ${ }^{6} \mathrm{CD}$ is a rare neoplasm of lymphatic origin that can be classified into two histological variants: a hyaline-vascular type, accounting for over $90 \%$ of cases, and the plasma cell type, which is far less common, as well as intermediate types. ${ }^{7}$ The hyaline-vascular type usually presents with a localized mass and is benign, whereas the histology of the plasma cell type is associated with multicentric disease taking a progressive course. Although most patients with the benign type of $\mathrm{CD}$ have shown remission of PNP upon excision of the tumour, ${ }^{8}$ our patient showed no improvement, possibly because of the occurrence of severe pulmonary involvement. Pulmonary involvement in PNP is common, and is the most frequent cause of death; for instance, Nikolskaia et al. ${ }^{9}$ have recently reported pulmonary involvement in 26 of 28 patients with CD and PNP. Respiratory failure results possibly from sloughing of the bronchial epithelium and its aspiration, with subsequent obstruction of small airways. ${ }^{2}$ As for the mechanisms by which CD induces PNP, it has been proposed that autoantibodies secreted from the Castleman tumour itself may play a pivotal role. ${ }^{10}$ This hypothesis seems to be supported in particular by the present case, in which CD may have triggered both the FDC sarcoma and the PNP.
Institute of Dermatological Sciences,

University of Milan,

IRCCS Ospedale Maggiore of Milan,

via Pace 9, 20122 Milan, Italy

*Molecular and Cell Biology Laboratory,

Istituto Dermopatico dell'Immacolata,

IDI-IRCCS, Rome 00167, Italy

†University of Milan 'Bicocca', Milan, Italy

Correspondence: Pamela Vezzoli

E-mail: pamela.vezzoli@libero.it
A.V. Marzano

P. Vezzoli

F. Mariotti*

V. BONESCHI

R. Caputo

E. BERTI†
1 Anhalt GJ, Kim SC, Stanley JR et al. Paraneoplastic pemphigus: an autoimmune mucocutaneous disease associated with neoplasia. N Engl J Med 1990; 323:1729-35.

2 Nguyen VT, Ndoye A, Bassler KD et al. Classification, clinical manifestations and immunopathological mechanisms of the epithelial variant of paraneoplastic autoimmune multiorgan syndrome. Arch Dermatol 2001; 137:193-206.

3 Mahoney MG, Aho S, Uitto J, Stanley JR. The members of the plakin family of proteins recognized by paraneoplastic pemphigus antibodies include periplakin. J Invest Dermatol 1998; 111:30813.

4 Ishii K, Amagai M, Hall RP et al. Characterization of autoantibodies in pemphigus using antigen-specific enzyme-linked immunosorbent assay with baculovirus-expressed recombinant desmogleins. J Immunol 1997; 159:2010-17.

5 Lee IJ, Kim SC, Kim HS et al. Paraneoplastic pemphigus associated with follicular dendritic cell sarcoma arising from Castleman's tumor. J Am Acad Dermatol 1999; 40:294-7.

6 Chan ACL, Chan KW, Chan JKC et al. Development of follicular dendritic cell sarcoma in hyaline-vascular Castleman's disease of the nasopharynx: tracing its evolution by sequential biopsies. Histopathology 2001; 38:510-18.

7 Keller AR, Hochholzer L, Castleman B. Hyaline-vascular and plasma cell types of giant lymph node hyperplasia of the mediastinum and other locations. Cancer 1972; 29:670-83.

8 Hsiao CJ, Hsu MML, Lee JYY et al. Paraneoplastic pemphigus in association with a retroperitoneal Castleman's disease presenting with a lichen planus pemphigoides-like eruption. A case and review of literature. Br J Dermatol 2001; 144:372-6.

9 Nikolskaia OV, Nousari CH, Anhalt GJ. Paraneoplastic pemphigus in association with Castleman's disease. Br J Dermatol 2003; 149:1143-51.

10 Wang L, Bu D, Yang Y et al. Castleman's tumours and production of autoantibody in paraneoplastic pemphigus. Lancet 2004; 363 : 525-31.

Conflicts of interest: none declared. 